\title{
Approximate Bayesian Computation
}

\section{Phylogeography of montane grasshoppers}

Lacey Knowles studied grasshoppers in the genus Melanopus. She collected 1275bp of DNA sequence data from cytochrome oxidase I (COI) from 124 individuals of $M$. oregonensis and two outgroup speices. The specimens were collected from 15 "sky-island" sites in the northern Rocky Mountains (see Figure 1; [5]). Two alternative hypotheses had been proposed to describe the evolutionary relationships among these grasshoppers (refer to Figure 2 for a pictorial representation):

- Widespread ancestor: The existing populations might represent independently derived remnants of a single, widespread population. In this case all of the populations would be equally related to one another.

- Multiple glacial refugia: Populations that shared the same refugium will be closely related while those that were in different refugia will be distantly related.

As is evident from Figure 2, the two hypotheses have very different consequences for the coalescent history of alleles in the sample. Since the interrelationships between divergence times and time to common ancestry differ so markedly between the two scenarios, the pattern of sequence differences found in relation to the geographic distribution will differ greatly between the two scenarios.

Using techniques described in Knowles and Maddison [6], Knowles simulated gene trees under the widespread ancestor hypothesis. She then placed them within a population tree representing the multiple glacial refugia hypothesis and calculated a statistic, $s$, that measures the discordance between a gene tree and the population tree that contains it. This gave her a distribution of $s$ under the widespread ancestor hypothesis. She compared the $s$ estimated from her actual data with this distribution and found that the observed value of $s$ was only $1 / 2$ to $1 / 3$ the size of the value observed in her simulations. ${ }^{1}$ Let's unpack that a bit.

\footnotetext{
${ }^{1}$ The discrepancy was largest when divergence from the widespread ancestor was assumed to be very recent.

(C) 2010-2017 Kent E. Holsinger
} 


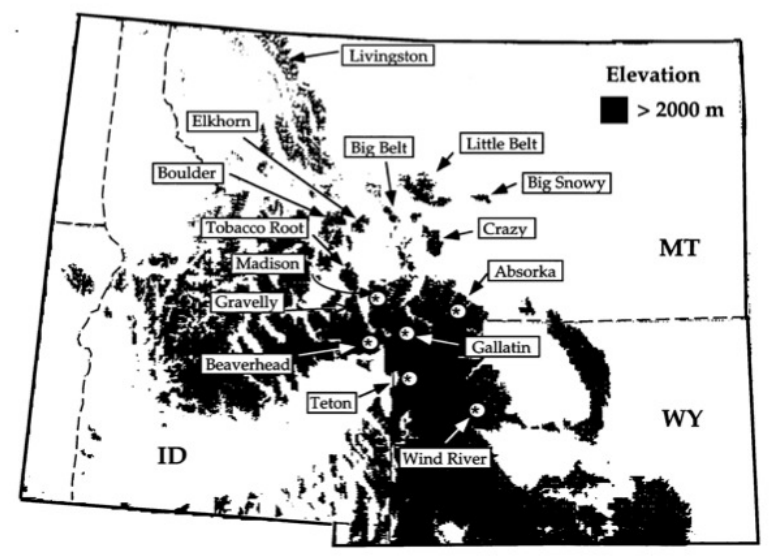

Figure 1: Collection sites for Melanopus oregonensis in the northern Rocky Mountains (from [5]).

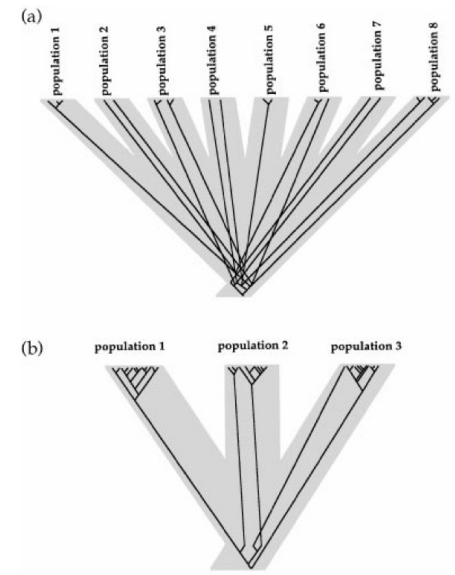

Figure 2: Pictorial representations of the "widespread ancestor" (top) and "glacial refugia" (bottom) hypotheses (from [5]). 
- Knowles estimated the the phylogeny of the haplotypes in her sample. $s$ is the estimated minimum number of among-population migration events necessary to account for where haplotypes are currently found given the inferred phylogeny [8]. Let's call the $s$ estimated from the data $s_{o b s}$.

- Then she simulated a neutral coalescence process in which the populations were derived from a single, widespread ancestral population. For each simulation she rearranged the data so that populations were grouped into separate refugia and estimated $s_{\text {sim }}$ from the rearranged data, and she repeated this 100 times for several different times since population splitting.

The results are shown in Figure 3. As you can see, the observed $s$ value is much smaller than any of those obtained from the coalescent simulations. That means that the observed data require far fewer among-population migration events to account for the observed geographic distribution of haplotypes than would be expected with independent origin of the populations from a single, widespread ancestor. In short, Knowles presented strong evidence that her data are not consistent with the widespread ancestor hypothesis.

\section{Approximate Bayesian computation: motivation}

Approximate Bayesian Computation ( $\mathrm{ABC}$ for short), extends the basic idea we've just seen to consider more complicated scenarios. The IMa approach developed by Nielsen, Wakely, and Hey is potentially very flexible and very powerful $[3,4,7]$. It allows for non-equilibrium scenarios in which the populations from which we sampled diverged from one another at different times, but suppose that we think our populations have dramatically increased in size over time (as in humans) or dramatically changed their distribution (as with an invasive species). Is there a way to use genetic data to gain some insight into those processes? Would I be asking that question if the answer were "No"?

\section{An example}

Let's change things up a bit this time and start with an example of a problem we'd like to solve first. Once you see what the problem is, then we can talk about how we might go about solving it. The case we'll discuss is the case of the cane toad, Bufo marinus, in Australia.

You may know that the cane toad is native to the American tropics. It was purposely introduced into Australia in 1935 as a biocontrol agent, where it has spread across an area of more than 1 million $\mathrm{km}^{2}$. Its range is still expanding in northern Australia and to a lesser 

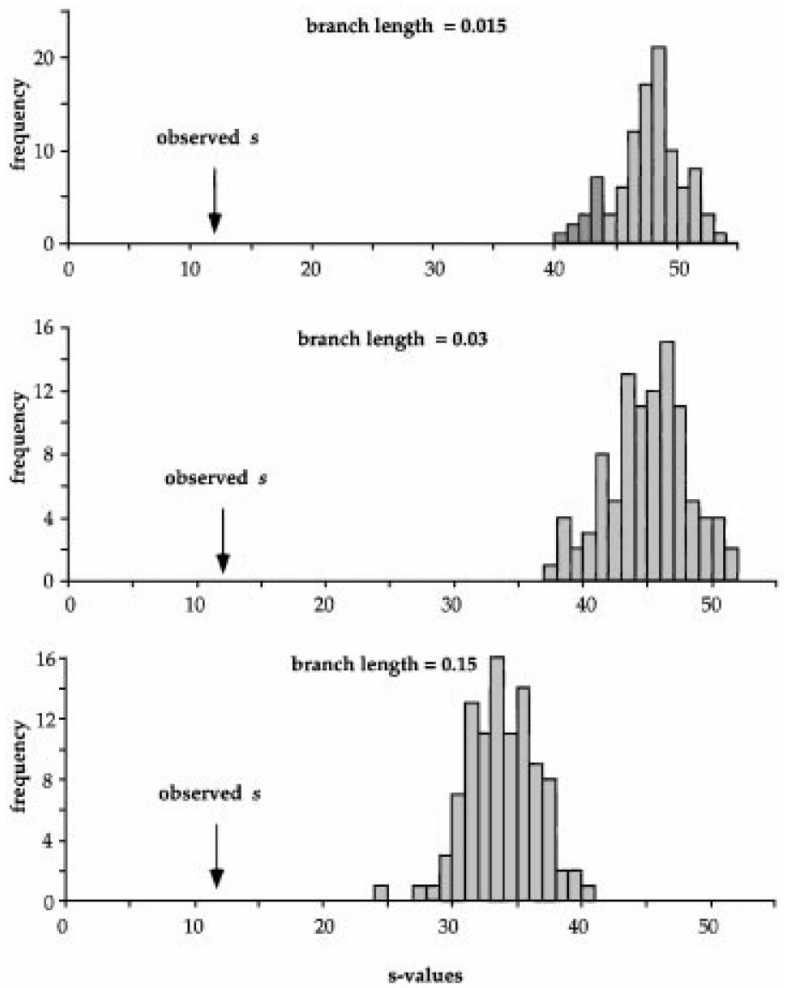

Figure 3: Distribution of the observed minimum number of among-population migration events, $s$, and the expected minimum number of migration events under the "widespread ancestor" hypothesis. (from [5]). 
extent in eastern Australia (Figure 4). ${ }^{2}$ Estoup et al. [2] collected microsatellite data from 30 individuals in each of 19 populations along roughly linear transects in the northern and eastern expansion areas.

With these data they wanted to distinguish among five possible scenarios describing the geographic spread:

- Isolation by distance: As the expansion proceeds, each new population is founded by or immigrated into by individuals with a probability proportional to the distance from existing populations.

- Differential migration and founding: Identical to the preceding model except that the probability of founding a population may be different from the probability of immigration into an existing population.

- "Island" migration and founding: New populations are established from existing populations without respect to the geographic distances involved, and migration occurs among populations without respect to the distances involved.

- Stepwise migration and founding with founder events: Both migration and founding of populations occurs only among immediately adjacent populations. Moreover, when a new population is established, the number of individuals involved may be very small.

- Stepwise migration and founding without founder events: Identical to the preceding model except that when a population is founded its size is assumed to be equal to the effective population size.

That's a pretty complex set of scenarios. Clearly, you could use Migrate or IMa2 to estimate parameters from the data Estoup et al. [2] report, but would those parameters allow you to distinguish those scenarios? Not in any straightforward way that I can see. Neither Migrate nor IMa2 distinguishes between founding and migration events for example. And with IMa2 we'd have to specify the relationships among our sampled populations before we could make any of the calculations. In this case we want to test alternative hypotheses of population relationship. So what do we do?

\footnotetext{
${ }^{2}$ All of this information is from the introduction to [2].
} 


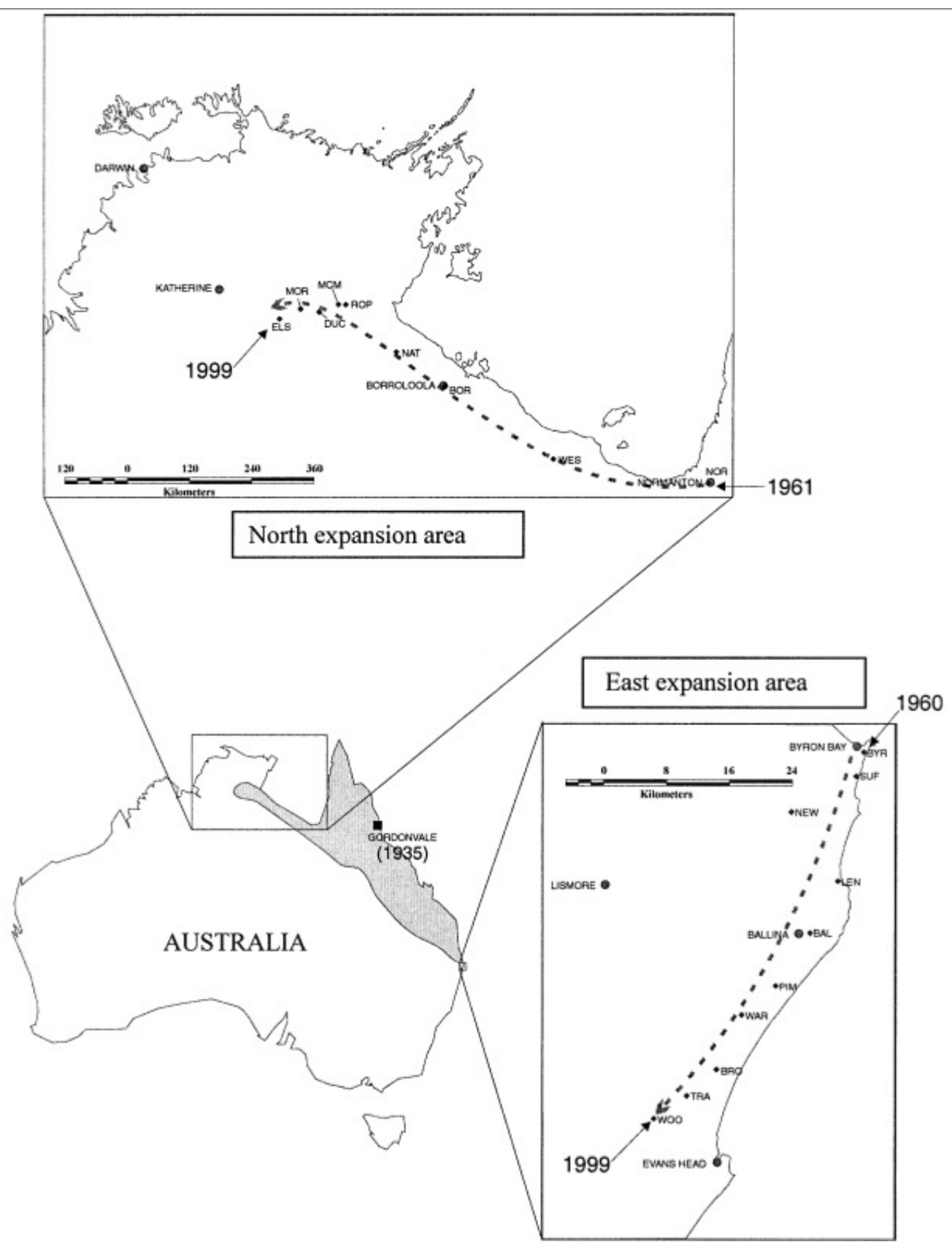

Figure 4: Maps showing the expansion of the cane toad population in Australia since its introduction in 1935 (from [2]). 


\section{Approximate Bayesian Computation}

Well, in principle we could take an approach similar to what Migrate and IMa2 use. Let's start by reviewing what we did last time ${ }^{3}$ with Migrate and IMa2. In both cases, we knew how to simulate data given a set of mutation rates, migration rates, local effective population sizes, and times since divergence. Let's call that whole, long string of parameters $\xi$ and our big, complicated data set $X$. If we run enough simulations, we can keep track of how many of those simulations produce data identical to the data we collected. With those results in hand, we can estimate $P(X \mid \xi)$, the likelihood of the data, as the fraction of simulations that produce data identical to the data we collected. ${ }^{4}$ In principle, we could take the same approach in this, much more complicated, situation. But the problem is that there are an astronomically large number of different possible coalescent histories and different allelic configurations possible with any one population history both because the population histories being considered are pretty complicated and because the coalescent history of every locus will be somewhat different from the coalescent history at other loci. As a result, the chances of getting any simulated samples that match our actual samples is virtually nil, and we can't estimate $P(X \mid \xi)$ in the way we have so far.

Approximate Bayesian computation is an approach that allows us to get around this problem. It was introduced by Beaumont et al. [1] precisely to allow investigators to get approximate estimates of parameters and data likelihoods in a Bayesian framework. Again, the details of the implementation get pretty hairy, ${ }^{5}$ but the basic idea is relatively straightforward. ${ }^{6}$

1. Calculate "appropriate" summary statistics for your data set, e.g., pairwise estimates of $\phi_{S T}$ (possibly one for every locus if you're using microsatellite or SNP data), estimates of within population diversity, counts of the number of segregating sites (for nucleotide sequence data, both within each population and across the entire sample) or counts of the number of segregating alleles (for microsatellite data). Call that set of summary statistics $S$.

2. Specify a prior distribution for the unknown parameters, $\xi$.

\footnotetext{
${ }^{3}$ More accurately, what Peter Beerli, Joe Felsenstein, Rasmus Nielsen, John Wakeley, and Jody Hey did.

${ }^{4}$ The actual implementation is a bit more involved than this, but that's the basic idea.

${ }^{5}$ You're welcome to read the Methods in [1], and feel free to ask questions if you're interested. I have to confess that there's a decent chance I won't be able to answer your question until I've done some further studying. I've only used ABC a little, and I haven't used it for anything that I've published - yet.

${ }^{6} \mathrm{OK}$. This maybe calling it "relatively straightforward" is misleading. Even this simplified outline is fairly complicated, but compared to some of what you've already survived in this course, it may not look too awful.
} 
3. Pick a random set of parameter values, $\xi^{\prime}$ from the prior distribution and simulate a data set for that set of parameter values.

4. Calculate the same summary statistics for the simulated data set as you calculated for your actual data. Call that set of statistics $S^{\prime}$.

5. Calculate the distance between $S$ and $S^{\prime} .{ }^{7}$ Call it $\delta$. If it's less than some value you've decided on, $\delta^{*}$, keep track of $S^{\prime}$ and the associated $\xi^{\prime}$ and $\delta$. Otherwise, throw all of them away and forget you ever saw them.

6. Return to step 2 and repeat until you you have accepted a large number of pairs of $S^{\prime}$ and $\xi^{\prime}$.

Now you have a bunch of $S^{\prime}$ s and a bunch of $\xi^{\prime}$ s that produced them. Let's label them $S_{i}$ and $\xi_{i}$, and let's remember what we're trying to do. We're trying to estimate $\xi$ for our real data. What we have from our real data is $S$. So far it seems as if we've worked our computer pretty hard, but we haven't made any progress.

Here's where the trick comes in. Suppose we fit a regression to the data we've simulated

$$
\xi_{i}=\alpha+S_{i} \beta+\epsilon,
$$

where $\alpha$ is an intercept, $\beta$ is a vector of regression coefficients relating each of the summary statistics to $\xi$, and $\epsilon$ is an error vector. ${ }^{8}$ Once we've fit this regression, we can use it to predict what $\xi$ should be in our real data, namely

$$
\xi=\alpha+S \beta,
$$

where the $S$ here corresponds to our observed set of summary statistics. If we throw in some additional bells and whistles, we can approximate the posterior distribution of our parameters. With that we can get not only a point estimate for $\xi$, but also credible intervals for all of its components.

\footnotetext{
${ }^{7}$ You could use any one of a variety of different distance measures. A simple Euclidean distance might be useful, but you could also try something more complicated, like a Mahalanobis distance.

${ }^{8}$ I know what you're thinking to yourself now. This doesn't sound very simple. Trust me. It is as simple as I can make it. The actual procedure involves local linear regression. I'm also not telling you how to go about picking $\delta$ or how to pick "appropriate" summary statistics. There's a fair amount of "art" involved in that.
} 


\section{Back to the real world ${ }^{9}$}

OK. So now we know how to do ABC, how do we apply it to the cane toad data. Well, using the additional bells and whistles I mentioned, we end up with a whole distribution of $\delta$ for each of the scenarios we try. The scenario with the smallest $\delta$ provides the best fit of the model to the data. In this case, that corresponds to model 4, the stepwise migration with founder model, although it is only marginally better than model 1 (isolation by distance) and model 2 (isolation by distance with differential migration and founding) in the northern expansion area (Figure 5).

Of course, we also have estimates for various parameters associated with this model:

- $N_{e_{s}}$ : the effective population size when the population is stable.

- $N_{e_{f}}$ : the effective population size when a new population is founded.

- $F_{R}$ : the founding ratio, $N_{e_{s}} / N_{e_{f}}$.

- $m$ : the migration rate.

- $N_{e_{s}} m$ : the effective number of migrants per generation.

The estimates are summarized in Table 1. Although the credible intervals are fairly broad, ${ }^{10}$ there are a few striking features that emerge from this analysis.

- Populations in the northern expansion area are larger, than those in the eastern expansion region. Estoup et al. [2] suggest that this is consistent with other evidence suggesting that ecological conditions are more homogeneous in space and more favorable to cane toads in the north than in the east.

- A smaller number of individuals is responsible for founding new populations in the east than in the north, and the ratio of "equilibrium" effective size to the size of the founding population is bigger in the east than in the north. (The second assertion is only weakly supported by the results.)

- Migration among populations is more limited in the east than in the north.

As Estoup et al. [2] suggest, results like these could be used to motivate and calibrate models designed to predict the future course of the invasion, incorporating a balance between gene flow (which can reduce local adaptation), natural selection, drift, and colonization of new areas.

\footnotetext{
${ }^{9}$ Or at least something resembling the real world

${ }^{10}$ And notice that these are $90 \%$ credible intervals, rather than the conventional $95 \%$ credible intervals, which would be even broader.
} 
East expansion area (EEA)

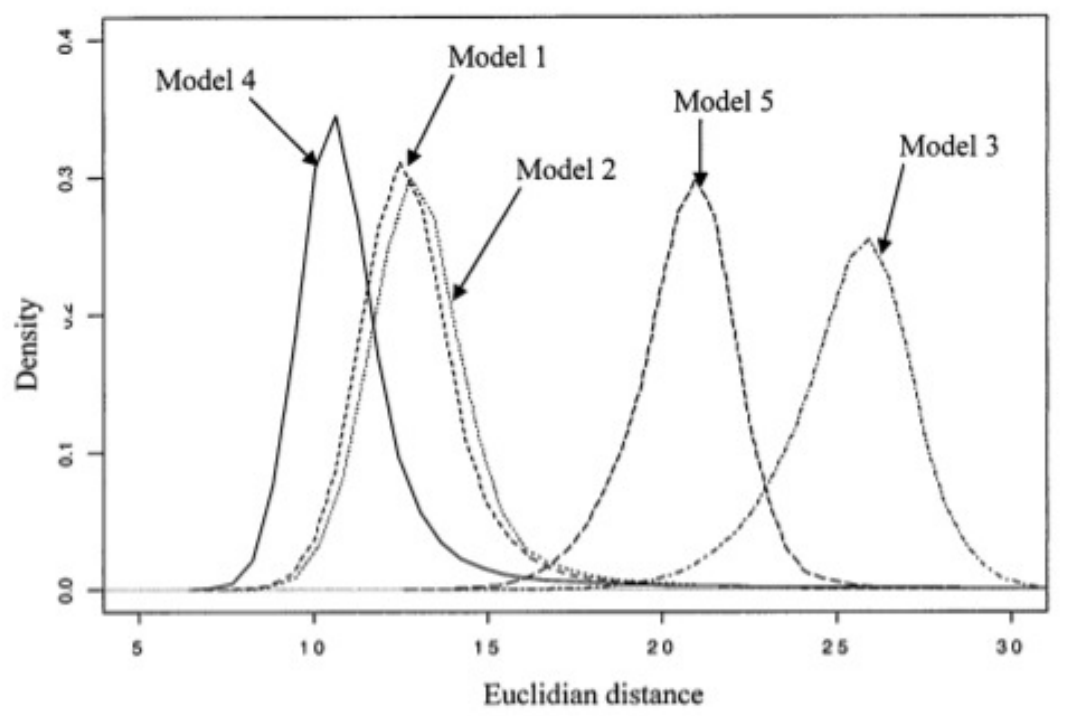

North expansion area (NEA)

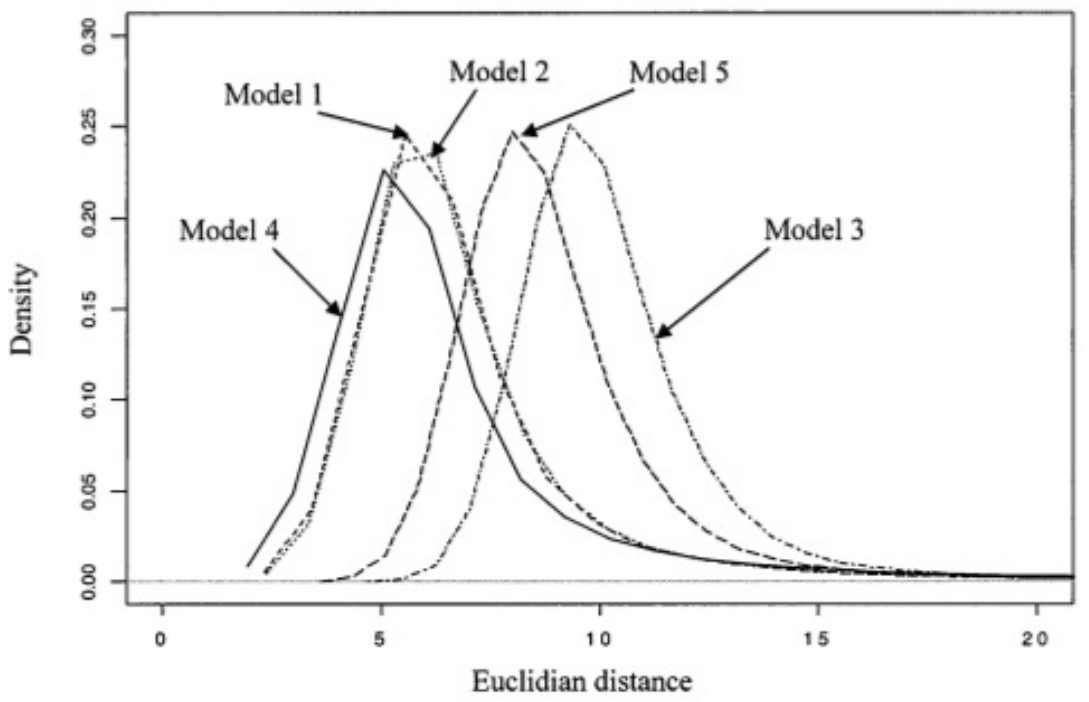

Figure 5: Posterior distribution of $\delta$ for the five models considered in Estoup et al. [2]. 


\begin{tabular}{ccr}
\hline \hline Parameter & area & mean $(5 \%, 90 \%)$ \\
\hline$N_{e_{s}}$ & east & $744(205,1442)$ \\
& north & $1685(526,2838)$ \\
$N_{e_{f}}$ & east & $78(48,118)$ \\
& north & $311(182,448)$ \\
$F_{R}$ & east & $10.7(2.4,23.8)$ \\
& north & $5.9(1.6,11.8)$ \\
$m$ & east & $0.014\left(6.0 \times 10^{-6}, 0.064\right)$ \\
& north & $0.117\left(1.4 \times 10^{-4}, 0.664\right)$ \\
$N_{e_{s}} m$ & east & $4.7(0.005,19.9)$ \\
& north & $188(0.023,883)$ \\
\hline
\end{tabular}

Table 1: Posterior means and 90\% credible intervals for parameters of model 4 in the eastern and northern expansion areas of Bufo marinus.

\section{Limitations of ABC}

If you've learned anything by now, you should have learned that there is no perfect method. An obvious disadvantage of ABC relative to either Migrate or IMa2 is that it is much more computationally intensive.

- Because the scenarios that can be considered are much more complex, it simply takes a long time to simulate all of the data.

- In the last few years, one of the other disadvantages - that you had to know how to do some moderately complicated scripting to piece together several different packages in order to run analysis - has become less of a problem. popABC (http: //code.google.com/p/popabc/ and DIYABC (http://www1.montpellier.inra.fr/ $\mathrm{CBGP} /$ diyabc/) make it relatively easy ${ }^{11}$ to perform the simulations.

- Selecting an appropriate set of summary statistics isn't easy, and it turns out that which set is most appropriate may depend on the value of the parameters that you're trying to estimate and the which of the scenarios that you're trying to compare is closest to the actual scenario applying to the populations from which you collected the data. Of course, if you knew what the parameter values were and which scenario was closest to the actual scenario, you wouldn't need to do ABC in the first place.

\footnotetext{
${ }^{11}$ Emphasis on "relatively".
} 
- In the end, $\mathrm{ABC}$ allows you to compare a small number of evolutionary scenarios. It can tell you which of the scenarios you've imagined provides the best combination of fit to the data and parsimonious use of parameters (if you choose model comparison statistics that include both components), but it takes additional work to determine whether the model is adequate, in the sense that it does a good job of explaining the data. Moreover, even if you determine that the model is adequate, you can't exclude the possibility that there are other scenarios that might be equally adequate - or even better.

\section{References}

[1] Mark A Beaumont, Wenyang Zhang, and David J Balding. Approximate Bayesian computation in population genetics, 2002.

[2] Arnaud Estoup, Mark A Beaumont, Florent Sennedot, Craig Moritz, and Jean-Marie Cornuet. Genetic analysis of complex demographic scenarios: spatially expanding populations of the cane toad, Bufo marinus, 2004.

[3] Jody Hey and Rasmus Nielsen. Multilocus methods for estimating population sizes, migration rates and divergence time, with applications to the divergence of Drosophila pseudoobscura and D. persimilis, 2004.

[4] Jody Hey and Rasmus Nielsen. Integration within the Felsenstein equation for improved Markov chain Monte Carlo methods in population genetics. Proceedings of the National Academy of Sciences, 104(8):2785-2790, 2007.

[5] L Knowles. Did the Pleistocene glaciations promote divergence? Tests of explicit refugial models in montane grasshopprers. Molecular Ecology, 10(3):691-701, 2001.

[6] L Knowles and Wayne P Maddison. Statistical phylogeography. Molecular Ecology, 11(12):2623-2635, 2002.

[7] Rasmus Nielsen and J Wakeley. Distinguishing migration from isolation: a Markov chain Monte Carlo approach, 2001.

[8] Montgomery Slatkin and Wayne Maddison. A cladistic measure of gene flow inferred from the phylogenies of alleles. Genetics, 123:603-613, 1989. 


\section{Creative Commons License}

These notes are licensed under the Creative Commons Attribution 4.0 International License (CC BY 4.0). To view a copy of this license, visit

http://creativecommons.org/licenses/by/4.0/ or send a letter to Creative Commons, 559 Nathan Abbott Way, Stanford, California 94305, USA. 